\title{
RNA: yesterday, today and tomorrow
}

\author{
VENKAT GOPALAN ${ }^{1}$ and WILLIAM H. MCCLAIN ${ }^{2}$ \\ ${ }^{1}$ Department of Chemistry and Biochemistry, and Center for RNA Biology, The Ohio State University, Columbus, Ohio 43210, USA \\ ${ }^{2}$ Department of Bacteriology, University of Wisconsin, Madison, Wisconsin 53706, USA
}

As part of celebrating the 20th anniversary of the RNA journal, we sought to identify an exemplar from recent advances in RNA research that integrates several fundamentals in molecular biology that have emerged since the genetic code was established 50 years ago. We then discuss an area where exploration in the near future will perhaps prove profitable for RNA biology.

\section{The atomic structures of the ribosome}

"The important thing is to be there when the picture is painted." J. Minton

It has been a long journey to decipher the roles of ribosomal RNAs (rRNAs), culminating in the stunning high-resolution crystallographic structures of the ribosome, both alone and bound to tRNAs and mRNA, notably from the pioneering work of Harry Noller, Venki Ramakrishnan, Thomas Steitz, and Ada Yonath. Few anticipated that the functional centers of both the 30S (the decoding site) and 50S (the peptidyl transferase center) would consist solely of RNA, making the ribosome an ancient ribozyme. Despite their mass being nearly equal to that of rRNAs, ribosomal proteins are relegated to scaffolding and chaperone functions. The atomic structures of the ribosome represent a major achievement that offered a grand scientific opening to the 21 st century and initiated new studies targeted towards a mechanistic understanding of the various steps in translation. Importantly, there is something for everyone in life sciences, as highlighted below with a small sampling.

First, lessons related to catalysis were acquired both directly from the crystal structures and indirectly from biochemical, genetic and computational studies. The ribosome performs two key reactions: synthesis of polypeptides from aminoacyl-tRNAs and hydrolysis to release the polypeptide end product, although the latter requires auxiliary factors. The first act, ester aminolysis, is surprisingly driven largely by entropic features and not through conventional general

\footnotetext{
Corresponding authors: gopalan.5@osu.edu; wmcclain@wisc.edu Article and publication date are at http://www.rnajournal.org/cgi/doi/ 10.1261/rna.049759.115. Freely available online through the RNA Open Access option.
}

acid/base catalysis of highly conserved residues in the active site. The ribosome offers a pre-organized hydrogen bonding network and an electrostatic environment that enhances peptide bond formation $10^{6}$-fold compared to the uncatalyzed reaction through concerted participation of the rRNA active site, the substrate tRNA, and several water molecules. Such catalytic requirements may have been fulfilled in part by RNA world primitive ribosomes, whose polypeptide products ultimately championed the evolution of the more complex extant version.

Second, these structures also provided answers to longstanding questions. For example, the accurate discrimination between cognate and non-cognate tRNAs was long suspected to result not merely from the thermodynamic differences between correct and incorrect base pairs in codon (mRNA)-anticodon (tRNA) interactions. The crystal structures revealed the elegant hand of the ribosome in decoding accuracy: a conformational change is effected for downstream steps only after interrogation of the codon-anticodon interaction, with the ribosome strictly restricting the first two positions to Watson-Crick base pairs while allowing some flexibility at the third position clearly accommodating the wobble (non-canonical) pairs. The energy gained from these interactions is used to ensure decoding fidelity, a vital aspect in transfer of genetic information in all biological organisms. Similarly, it is now evident how limited structural differences between eukaryotic (mitochondrial variant included) and prokaryotic ribosomes provide the underpinning for the efficacy and selectivity of antibiotics in blocking microbial translation. Understanding the structural basis of resistance and susceptibility is essential to develop new and repurposing existing antibiotics, a goal whose importance cannot be overstated.

Last, the tour de force of obtaining an atomic-level picture of the ribosome has both upped the ante and is a source of inspiration for questions one might not have been emboldened to pursue previously (in translation and elsewhere). Even as a watchmaker's virtuosity is evidenced only upon

(C) 2015 Gopalan and McClain This article, published in RNA, is available under a Creative Commons License (Attribution-NonCommercial 4.0 International), as described at http://creativecommons.org/licenses/by-nc/4.0/. 
seeing the inner machinations of the timepiece, the ribosome's molecular choreography is being slowly parsed out thanks to snapshots obtained from crystallography and cryo-EM. These studies of pre- and post-reaction states and intermediate states of translocation reveal the intricate motions of the two ribosomal subunits with respect to each other during the flow of tRNAs through the massive ribosome workbench. The two subunits function coordinately as a molecular ratchet to synchronize the movement of tRNAs and mRNA to maintain the reading frame. These insights on the smooth cooperation between different parts of an asymmetric RNA-based molecular machine will be instructive to the nascent field of RNA tectonics that aims to design dynamic RNA nanostructures for bioengineering.

\section{Deciphering the connection between cellular metabolism and non-coding RNA function}

"In research the front line is almost always in a fog." F. H. C. Crick

A comprehensive inventory encompassing the "where," "when" and "why" of RNA modifications is the first step in this next frontier linking cellular metabolism and RNA biology. Modified nucleotides are formed post-transcriptionally in Archaea, Bacteria, and Eukarya, and are present in rRNAs, tRNAs, small nuclear RNAs, tmRNA, spliceosomal RNAs, and mRNAs. About 110 distinct (base and ribose) modifications are known to exist in RNA; notably, there are no reports of modified phosphates in RNA. This inventory to date excludes changes resulting from RNA editing, another fascinating chapter in RNA biology. Nearly 160 enzymes bring about these RNA modifications, with crystal structures now available for more than 50 of them.

RNA modifications, which can be highly conserved, entail a high genetic cost given the need to synthesize specialized modification enzymes. In addition to expanding the basic quadruple RNA alphabet, this layer of complexity diversifies RNA structure and function as illustrated here with two examples. The first is dihydrouracil (D), a uracil derivative that is present in several positions in the D loop of tRNAs and for which the $\mathrm{D}$ arm was named. While nucleic acid bases are flat and pack in oligonucleotide structures by stacking interactions, D is fully saturated and not planar. Thus, D disturbs packing interactions, thereby altering local conformation and global structure. In tRNAs, the D residues confer conformational flexibility, which facilitate the universally conserved tertiary contacts between the $\mathrm{D}$ and $\mathrm{T}$ loops and lead to dynamic motions that extend into structures adjoining the D loop. Interestingly, Robert Holley's pioneering efforts in 1965 to determine the sequence of yeast tRNA ${ }^{\mathrm{Ala}}$ were expedited by the distinctive chemical and fractionation properties of D that facilitated ordering of the overlapping oligonucleotides obtained by partial digestion of the intact tRNA with ribonucleases.
The second modified nucleotide exemplified here is the wobble nucleotide in the anticodon of a microbial tRNA ${ }^{\text {Ile }}$ where the $\mathrm{O} 2$ of cytidine 34 is replaced by lysine giving rise to lysidine (L34). This happy marriage of a nucleobase and an amino acid remarkably alters both the codon recognition properties of the tRNA from AUG to AUA and the amino acid acceptor specificity from methionine to isoleucine. Lysidine base pairs specifically with adenine in the wobble position of AUA and not with guanine in AUG, and thus imparts translational fidelity. The significance of this exquisite modification system is also supported by the observation that T4 bacteriophage encodes a similar tRNA ${ }^{\text {Ile }}$ which, unlike the other tRNAs present in its genome, is essential for replication in some Escherichia coli host strains.

These two examples of nucleotide modifications show that unadorned RNA transcripts can mask structural and functional attributes that surface only with RNA modifications. While the widespread use of RNA transcripts produced in vitro has greatly accelerated biochemical and biophysical investigations that require large amounts of target RNAs, obtaining RNAs that mirror the in vivo post-transcriptional decorations should be a high priority to gain an accurate and true picture of the design and function of biological systems. RNA modifications also likely play a broader regulatory role. Through a network of decisions, a principal goal of metabolic regulation is to couple growth with nutrient availability while ensuring organismal fitness. Within this central rubric, many complex choices and tradeoffs are made (e.g., to indulge in metabolic efficiency despite catalytic inefficiency or vice versa). Covalent modification (e.g., phosphorylation) is widely employed as a regulatory mechanism to rapidly change the activity of protein enzymes in metabolic pathways in response to alterations in nutrient/energy-charge levels. Similarly, changing the function of the large repertoire of cellular RNAs and RNPs through such means seems likely given the chemically diverse palette of RNA modifications that depend on the availability of various metabolites (e.g., sugar phosphates, acetyl-CoA, pyruvate, amino acids).

Recent studies strongly support tRNA and rRNA modifications as part of a strategy at times of duress to curtail cost-intensive translation, either globally or in a restricted fashion entailing subsets of mRNAs with specific codons, supporting the idea that RNA modifications are ideal prospects as sentries to regulate cellular growth. However, the nexus between cellular metabolism and RNA function might encompass all non-coding RNAs and function as part of the normal regulatory landscape (i.e., not restricted to stress conditions). If so, extant metabolism and the underlying regulatory web are both likely to be a palimpsest from the RNA world. Support for this notion could come from two approaches: (1) finding ribozymes that modify RNA to exert functional changes, an anticipation prompted by the array of metabolites bound by riboswitches, and (2) mapping system-wide alterations in RNA function as a result of modifications spurred by physiological cues. All macromolecular 
modifications entail writers, erasers and readers-elucidating the sequence and combinatorial scope of these players and knowing the context where specific RNA modifications engender schizophrenic lives of an RNA may help uncover the basis of diseases. This expectation is supported by various findings, including the association between encephalopathies and pathogenic mutations in human mitochondrial tRNAs that affect modifications. An altered tRNA methyltransferase activity has also been identified as a cause of type 2 diabetesfailure to fully modify tRNA ${ }^{\text {Lys }}$ in pancreatic $\beta$-cells leads to decreased translational accuracy and a reduction in pro-insulin synthesis.

\section{Envoi}

Thematic parallels (e.g., the chemiosmotic theory) in all organisms offer the strongest support for the theory of evolu- tion. An increased appreciation of RNA's biological versatility may lead to the inevitable conclusion, however, that there is plurality rather than conservation in how RNAs form the dynamic systems that bring each cell to life and that such biological innovation-inspired idiosyncrasies afford robustness in unique niches. Were both the rise and fall of the RNA empire then attributable to the remarkable resourcefulness of RNAs, which enabled stochastic excursions between divergent regulatory and functional strategies? New genome editing and genetic retrofitting methods hold promise for aiding in this quest to understand evolution.

\section{Acknowledgments}

Research in V.G.'s laboratory is supported by a grant from the National Science Foundation (MCB-0843543). 

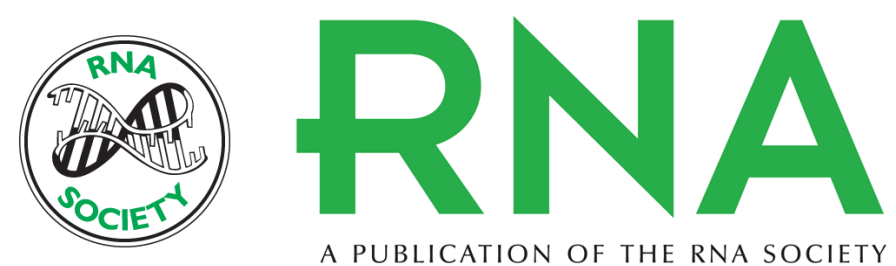

A PUBLICATION OF THE RNA SOCIETY

\title{
RNA: yesterday, today and tomorrow
}

\author{
Venkat Gopalan and William H. McClain
}

RNA 2015 21: 541-543

Open Access Freely available online through the RNA Open Access option.

Creative This article, published in RNA, is available under a Creative Commons License

Commons (Attribution-NonCommercial 4.0 International), as described at

License http://creativecommons.org/licenses/by-nc/4.0/.

Email Alerting Receive free email alerts when new articles cite this article - sign up in the box at the Service top right corner of the article or click here. 